\title{
Nephrometry Scores: The Effect of Imaging on Routine Read-out and Prediction of Outcome of Nephron-sparing Surgery
}

\author{
JULA WADLE ${ }^{1}$, SVETLANA HETJENS $^{2}$, JUDITH WINTER ${ }^{3}$, JULIA MÜHLBAUER ${ }^{1}$, MANUEL NEUBERGER $^{1}$, \\ FRANK WALDBILLIG ${ }^{1}$, FREDERIK WESSELS ${ }^{1}$, NIKLAS WESTHOFF ${ }^{1}$, PHILIPP RIFFEL $^{3}$, \\ NINA WAGENER ${ }^{1}$, PATRICK HONECK $^{1 *}$ and MAXIMILIAN CHRISTIAN KRIEGMAIR ${ }^{1 *}$ \\ Departments of ${ }^{1}$ Urology, and ${ }^{3}$ Radiology and Nuclear Medicine, \\ University Medical Centre Mannheim, Mannheim, Germany; \\ ${ }^{2}$ Department of Medical Statistics, Medical Faculty Mannheim, University of Heidelberg, Mannheim, Germany
}

\begin{abstract}
Background/Aim: This study investigated the impact of available preoperative imaging on the reliability and predictive accuracy of RENAL and PADUA nephrometry-scoring systems for renal tumors. Patients and Methods: Five urologists determined RENAL and PADUA scores using preoperative imaging data (computed tomography and magnetic resonance imaging) of 100 patients admitted for partial nephrectomy with the following combinations: T0: transverse planes without excretory phase (EP), TCO: transverse and coronal planes without EP, TC1: transverse and coronal planes with EP. Reference standard was obtained by a uro-radiologist. Ischemia time was used as surrogate for surgical complexity. Results: Assignment of EP significantly reduced interobserver-variability among urologists $(p<0.0001)$. Predictive accuracy for surgical complexity or correct assignment to nephrometry risk groups did not depend on image planes or EP. Conclusion: Interobserver variability, but not predictive accuracy of nephrometric systems, is affected by additional usage of EP.
\end{abstract}

When technically feasible, nephron-sparing surgery (NSS) is the preferred surgical treatment for localized renal masses (1). A detailed understanding of anatomic characteristics of the renal mass is essential for preoperative planning. To provide objective prediction of surgical complexity, as well

\footnotetext{
*These Authors contributed equally to this study.

Correspondence to: Jula Wadle, University Medical Centre Mannheim, Department of Urology, Theodor-Kutzer-Ufer 1-3, 68167 Mannheim, Germany. Tel: +49 15789036716, e-mail: wadle@stud.uni-heidelberg.de
}

Key Words: Excretory phase, nephrometry scores, PADUA, partial nephrectomy, RENAL. as improve comparability of cohorts, standardized reporting is necessary. Therefore, multiple nephrometry scoring systems have been recently published, of which RENAL (radius, exophytic/endophytic properties, nearness of tumor to the collecting system or sinus, anterior/posterior, location relative to polar lines) and PADUA (preoperative aspects and dimensions used for anatomical classification) are most established (2). They allow stratification of renal tumors into 'low-risk', 'intermediate-risk' and 'high-risk' groups (3, 4). The scores show good correlation with ischemia time (IT) during surgery, and can predict perioperative, functional, and oncological outcome of NSS (5-8). Thus, they may assist in clinical decision-making (9). Generally, RENAL and PADUA are determined from cross-sectional imaging. The recommended technique for primary imaging and staging in the diagnosis of renal cell carcinoma is contrast-enhanced abdominal computed tomography (CT) or magnetic resonance imaging (MRI) (1). A combination of unenhanced images, corticomedullary-phase images, and nephrographic-phase images provides highest diagnostic confidence but may fail to delineate the relationship between the renal mass and the urinary collecting system (UCS). However, involvement of the UCS greatly affects surgical complexity and is an essential part of the RENAL and PADUA systems. Depiction of proximity to or infiltration of the renal collecting system is best visualized on excretory-phase (EP) images $(10,11)$. Since the EP is not regularly included in primary imaging before NSS, this harbors a potential bias in determining nephrometric parameters for clinical and scientific purposes. Despite these known irregularities, there is no recommendation for a standard imaging data set that should be used when RENAL and PADUA are assessed. This makes comparability of studies difficult and reduces the validity and reproducibility of RENAL and PADUA.

The purpose of our study was to assess the impact of different cross-sectional imaging modalities (CT/MRI) as 
Table I. Characteristics of patients admitted for partial nephrectomy $(n=100)$.

\begin{tabular}{|c|c|c|c|c|}
\hline Characteristic & All $(n=100)$ & $\mathrm{CT}(\mathrm{n}=50)$ & MRI $(n=50)$ & $p$-Value \\
\hline Median patient age (range), years & $62(21-83)$ & $66(21-83)$ & $59(32-77)$ & 0.148 \\
\hline Gender, male, n (\%) & $64(64)$ & $32(64)$ & $32(64)$ & $>0.99$ \\
\hline Solitary kidney, n (\%) & $2(2)$ & $1(2)$ & $1(2)$ & $>0.99$ \\
\hline Median pathological tumor size (range), $\mathrm{cm}$ & $3(0.7-10.5)$ & $3.1(1.4-10.5)$ & $2.5(0.7-8)$ & 0.016 \\
\hline Malignant tumor, n (\%) & $76(76)$ & $39(78)$ & $37(74)$ & 0.64 \\
\hline \multicolumn{5}{|l|}{ Surgical approach } \\
\hline OPN, n (\%) & $90(90)$ & $44(88)$ & $46(92)$ & 0.505 \\
\hline RPN, n (\%) & $10(10)$ & $6(12)$ & $4(8)$ & \\
\hline Median operative time (IQR), min & $139(51-417)$ & $143(51-280)$ & $136(68-417)$ & 0.46 \\
\hline Intraoperative ischemia, $\mathrm{n}(\%)$ & $78(78)$ & $42(84)$ & $36(72)$ & 0.118 \\
\hline Median IT, min (IQR) & $17(4-41)$ & $16(10-36)$ & $18(4-41)$ & 0.357 \\
\hline Positive surgical margin, $\mathrm{n}(\%)$ & $2(2)$ & $1(2)$ & $1(2)$ & $>0.99$ \\
\hline Occurrence of complications*, n (\%) & $20(20)$ & $12(24)$ & $8(16)$ & 0.556 \\
\hline
\end{tabular}

CT: Computed tomography; IQR: interquartile range; MRI: magnetic resonance imaging; OPN: Open partial nephrectomy; RPN: robotic-assisted partial nephrectomy. $*>$ Clavien grade II.

well as the value of single sectional planes with and without EP for nephrometry scoring. It was our aim to establish a standard for image data required for valid and reproducible assessment of nephrometry scores to unify their scientific and clinical application.

\section{Patients and Methods}

Study design and radiological imaging data. After institutional Ethics Committee approval (2013-830-MA), 100 consecutive patients were identified with suitable preoperative imaging admitted between 2012 and 2016 for open partial nephrectomy or robotic assisted partial nephrectomy at the Department of Urology of the University Medical Centre Mannheim. Criteria for study inclusion were defined by an imaging protocol providing coronal and transverse planes, as well as unenhanced, corticomedullary-phase, nephrographic-phase, and EP images. Furthermore, all EP images were required to delineate the upper renal collecting system. Maximum slice thickness was not allowed to exceed $5 \mathrm{~mm}$ and MR images were to be derived from at least $1.5 \mathrm{~T}$ MRI. After standardized training, five urologists determined RENAL and PADUA independently for all patients by first considering only transverse planes without EP (T0). Thereafter, all cases were evaluated using transverse and coronal planes without EP (TC0). Finally, the combination of transverse and coronal planes with EP was assessed by the readers (TC1). To avoid learning and repetition effects, imaging was randomly displayed to the readers. A radiologist with experience in urological imaging determined RENAL and PADUA from TC1 to provide a reference standard.

Surgical parameters. Based on medical charts, patient characteristics, histopathological findings, and perioperative parameters were collected. Warm ischemia was used in all operations. Complications appearing up to 30 days after the operation were assessed using the Clavien classification in accordance to the European Association of Urologists Guidelines Panel Assessment and Recommendation (12, 13). All renal masses were categorized according to the nephrometry scoring systems RENAL and PADUA.
Statistical analysis. Quantitative variables are presented as median with range. Logistic regression was performed as a multivariate model to analyze two factors for a binary outcome simultaneously. Spearman's correlation (@) was used to determine interobserver variability (IOV) between urologists, as well as between urologists and the radiologist, for different sectional imaging planes separately. The results were then compared using the Wilcoxon signed-rank test. Furthermore, the correlation of the scores with IT was determined using Spearman's Q. Receiver operating characteristic (ROC) curves were generated for RENAL and PADUA predicting a prolonged IT, and area under the curve (AUC) values of plane settings were compared using the ROC contrast test. The proportions of correctly classified and over/underestimated tumors were evaluated for all imaging planes in order to compare concordance of correct risk stratification. All statistical calculations were made with SAS software, release 9.3 (SAS Institute Inc., Cary, NC, USA). Statistical significance was set at $p<0.05$.

\section{Results}

Patient characteristics and surgical outcome. Demographic data of the study population and surgical outcome are given in Table I. There were no significant differences in clinical or surgical parameters in patients with preoperative CT and MRI imaging except for tumor size, which was smaller in patients with preoperative MRI (3.1 cm vs. $2.5 \mathrm{~cm}, p=0.016)$. Overall, intraoperative IT was used in $78 \%(\mathrm{n}=78)$ of the cases. Median warm IT was 17 (4-41) minutes. Positive surgical margins occurred in $2 \%(n=2)$, severe complications greater than Clavien II were observed in $20 \%(n=20)$.

Interobserver variability for urologists. No significant difference of interobserver agreement among urologists was found between CT and MRI for RENAL or PADUA (Table II). Usage of TC1 was significantly associated with higher rates of agreement between urologists (RENAL: TC1 vs. T0: $p<0.0001, \mathrm{TC} 1 v s$. TC0: $p<0.0001$, TC0 vs. T0: $p=0.016$; PADUA: TC1 vs. T0: $p<0.0001$, TC1 vs. TC0: $p<0.0001$, TC0 vs. T0: $p=0.43$ ). 
Wadle et al: Impact of Sectional Imaging on Routine Read-out of Nephrometry Scores

Table II. Interobserver variability between urologists as measured by Spearman's $\varrho$ based on method, plane setting and nephrometry score.

\begin{tabular}{cclll}
\hline & All & CT & MRI & $p$-Value \\
\hline $\begin{array}{l}\text { RENAL } \\
\text { T0 }\end{array}$ & 0.363 & 0.369 & 0.353 & 0.407 \\
TC0 & 0.358 & 0.311 & 0.395 & 0.284 \\
TC1 & 0.406 & 0.362 & 0.44 & 0.088 \\
PADUA & & & & \\
T0 & 0.349 & 0.31 & 0.382 & 0.634 \\
TC0 & 0.363 & 0.291 & 0.425 & 0.861 \\
TC1 & 0.384 & 0.317 & 0.434 & 0.831 \\
\hline
\end{tabular}

CT: Computed tomography; MRI: magnetic resonance imaging; T0: transverse planes without excretory phase (EP); TC0: transverse and coronal planes without EP; TC1: Transverse and coronal planes with EP.

Correctness of scores obtained by urologists. Agreement of the scores obtained by urologists compared to the standard determined by the radiologist is displayed in Table III. Both nephrometry scoring systems showed highest conformity between urologists and radiologist when the additional EP was used (TC1: RENAL: $p=0.877$; PADUA: $p=0.867$ ). However, the benefit was not statistically significant (RENAL: $p=0.15$, PADUA: $p=0.295$ ).

Impact on clinical prediction. Finally, we analyzed the impact of using different sectional imaging planes with and without EP on the prediction of the IT as surrogate for surgical complexity (Table IV). The correlation between the nephrometry score and IT was highest when the radiologist was the reader (RENAL $\varrho=0.39$, PADUA $\mathrm{Q}=0.47)$. Scores obtained by the urologists revealed the highest correlation with IT when EP was used (RENAL $\varrho=0.255$, PADUA $\varrho=0.246)$. Urologists using the additional EP (TC1) were found to have the highest AUC values in ROC analyses, as shown in Figure 1 (AUC: RENAL standard (radiologist) $=0.8, \mathrm{TC} 1=0.72, \mathrm{TC} 0=0.7$, $\mathrm{T} 0=0.71$; PADUA: standard (radiologist) $=0.8, \mathrm{TC} 1=0.71$, $\mathrm{TC} 0=0.69, \mathrm{~T} 0=0.69$ ). When comparing the different AUC values, the predictive accuracy of the scores obtained by the radiologist were significantly higher, but there was no significant difference between the urologists using sectional imaging with and without EP (RENAL R-TC1 $p=0.004, \mathrm{R}-\mathrm{TC} 0 p=0.002, \mathrm{R}-\mathrm{T} 0 p=0.009$; PADUA R-TC1 $p=0.002$, R-TC0 $p<0.001, \mathrm{R}-\mathrm{T} 0 p=0,002$ ) (Table V). To determine the correct risk stratification, the proportions of correctly classified and over-/underestimated tumors were analyzed. Allocation to risk groups did not depend on the planes and phases used by the urologist, and was similar for TC1, TC0 and T0.
Table III. Spearman's correlation of scores obtained by urologists with the standard obtained by radiologist according to imaging modality.

\begin{tabular}{lll}
\hline & \multicolumn{2}{c}{ Spearman's @ } \\
\cline { 2 - 3 } Imaging modality & RENAL & PADUA \\
\hline T0 & 0.833 & 0.822 \\
TC0 & 0.85 & 0.85 \\
TC1 & 0.877 & 0.867 \\
\hline
\end{tabular}

T0: Transverse planes without excretory phase (EP); TC0: transverse and coronal planes without EP; TC1: transverse and coronal planes with EP.

Table IV. Spearman's correlation of ischemia time with score according to imaging modality.

\begin{tabular}{lcc}
\hline & \multicolumn{2}{c}{ Spearman's @ } \\
\cline { 2 - 3 } Imaging modality & RENAL & PADUA \\
\hline Radiologist & 0.39180 & 0.47074 \\
T0 & 0.23995 & 0.22135 \\
TC0 & 0.23643 & 0.21929 \\
TC1 & 0.25516 & 0.24644 \\
\hline
\end{tabular}

T0: Transverse planes without excretory phase (EP); TC0: transverse and coronal planes without EP; TC1: transverse and coronal planes with EP.

\section{Discussion}

Nephrometry scoring systems have broad application in objectifying renal tumor anatomy, thus improving comparability of studies and reliably predicting outcome of NSS. Generally, nephrometry scores are determined using preoperative sectional imaging $(3,4)$. It is important to emphasize the requirement for a multiplane dataset to characterize renal masses (1). With multidetector CT and modern MRI, multiple planes are available for most patients. An EP is usually not performed for preoperative imaging of cortical renal masses and is consequently not available for all patients. To date, the impact of using different imaging modalities (CT vs. MRI) and the availability of an additional EP on the outcome of nephrometry score assessment remains unclear. Unfortunately, many studies lack information on the quality and comprehensiveness of the images used to assess nephrometry scores (3-9). This harbors the risk of underlying bias when comparing studies. Consequently, there is a need to standardize better the requirements for the extent of the image data used to determine nephrometry scores.

In recent studies, CT and MRI were both used to assess nephrometry scores, without further distinction $(3-5,9,14)$. In our analyses, the imaging modality had no significant impact on interobserver agreement among urologists (Table II). Therefore, both modalities seem to be suitable for 
A
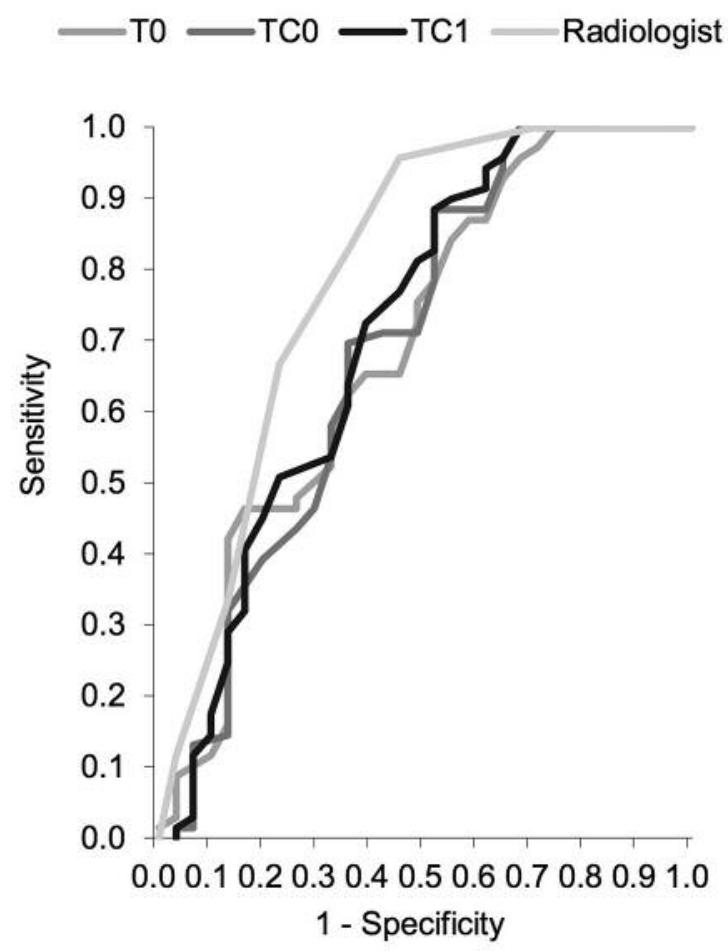

B

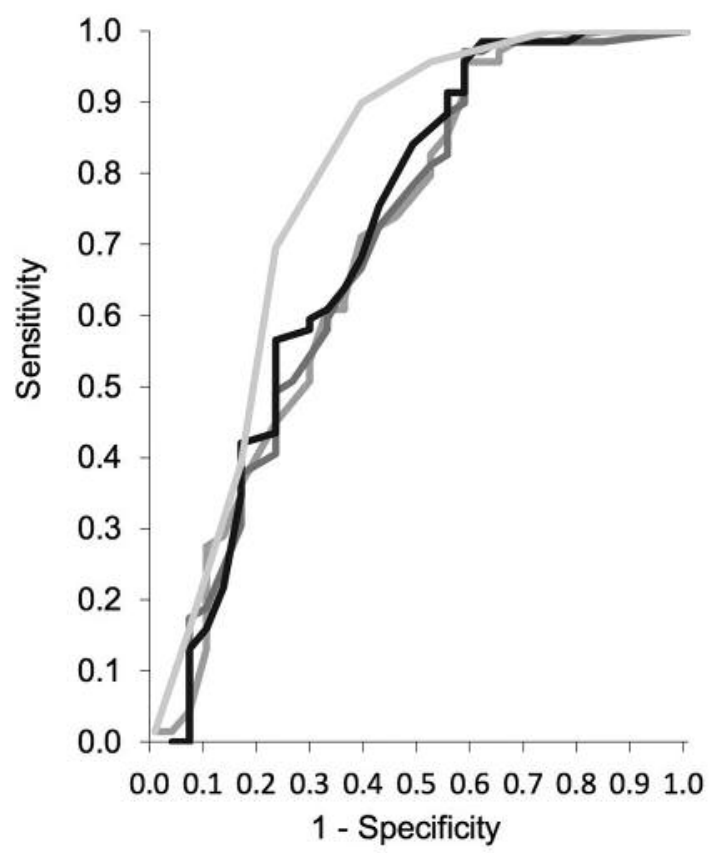

Figure 1. Receiver operating characteristics curves for correlation of radiologist and urologists for PADUA (A) and RENAL (B) scores for ischemia time. Area under the curve: A: Radiologist $=0.80, T C 1=0.72, T C 0=0.70, T 0=0.71 ; B:$ Radiologist $=0.80, T C 1=0.71, T C 0=0.69, T 0=0.69$.

assessing nephrometry scores and can be used in parallel. Remarkably, in our study, agreement between the radiologist and urologists outperformed findings reported in the latest literature, possibly due to the standardized training of the urologists prior to score assessment (14-16).

Our comprehensive analysis of the entire cohort revealed that the agreement among urologists was significantly improved when an additional EP is applied. Using an additional EP led to lowest IOV and high correlation with surgical complexity in TC1. This is clearly reflected by the main advantage of use of the EP, namely the detailed depiction of urinary tract anatomy (17). Thus, the European Association of Urology emphasizes the use of this modality because of its significantly high sensitivity and specificity for opacification of the urinary tract $(17,18)$.

In order to consolidate a possible recommendation to preferentially use images with EP for nephrometry score assessment, we tested the impact of using EP on the clinical validity of the scores. Here, IT was used as a clinical surrogate for surgical complexity $(2,19)$. In our cohort, the highest correlation between scores and IT was achieved using images with EP (TC1). However, the benefit was marginal. Furthermore, ROC analyses suggested that using
Table V. p-Values for Figure 1.

\begin{tabular}{|c|c|c|c|c|}
\hline RENAL PADUA & Radiologist & T0 & $\mathrm{TC} 0$ & $\mathrm{TC} 1$ \\
\hline Radiologist & & 0.002 & 0.0002 & 0.002 \\
\hline T0 & 0.009 & & 0.973 & 0.275 \\
\hline TC0 & 0.002 & 0.958 & & 0.047 \\
\hline $\mathrm{TC} 1$ & 0.004 & 0.441 & 0.103 & \\
\hline
\end{tabular}

T0: Transverse planes without excretory phase (EP); TC0: transverse and coronal planes without EP; TC1: transverse and coronal planes with EP.

the EP for score assessment has no significant benefit on the predictive accuracy of the scores. Finally, we compared the impact of using the EP on the agreement of nephrometry score risk stratification to the standard obtained by the radiologist (RENAL low complexity 4-6, intermediate complexity 7-9, high complexity 10-12; PADUA low complexity 6-7, intermediate complexity 8-9, high complexity $\geq 10)(3,4)$. Stratification to risk subgroups was similar for different sectional imaging data sets. Although the IOV in obtaining nephrometry scores between urologists can be reduced when an additional EP is used, its clinical 
impact seems to be negligible. The scores did not gain higher predictive accuracy and precision in risk stratification seems to be insignificant.

There are several limitations to our study. First and foremost, data from a single institution was analyzed and scores were assessed retrospectively. Furthermore, a reference standard was obtained by one radiologist only. However, interobserver agreement of radiologists has recently been shown to be excellent (20). Additionally, multiple CT and MRI scanners were utilized, although standard requirements were demanded for study inclusion of the respective patient.

\section{Conclusion}

This study provides the first comprehensive evaluation of imaging modalities and the use of EP on IOV and clinical validity of nephrometry scoring systems. Our findings suggest that the routine read-out of nephrometry scores by urologists does not differ using CT or MRI. Assessment of an additional EP may reduce IOV, leading to more consistent results. However, using the EP had no effect on the predictive accuracy of the surgical complexity or risk-stratification in our cohort. Therefore, our data suggest a two-plane dataset of either CT or MRI without EP to be sufficient for routine readout of nephrometry scores by urologists.

\section{Competing Interests}

The Authors declare that they have no competing interests.

\section{References}

1 Ljungberg B, Bensalah K, Canfield S, Dabestani S, Hofmann F, Hora M, Kuczyk MA, Lam T, Marconi L, Merseburger AS, Mulders P, Powles T, Staehler M, Volpe A and Bex A: EAU guidelines on renal cell carcinoma: 2014 update. Eur Urol 67: 913-924, 2015.

2 Hou W, Yan W and Ji Z: Anatomic features involved in technical complexity of partial nephrectomy. Urology 85: 1-7, 2015.

3 Ficarra V, Novara G, Secco S, Macchi V, Porzionato A, De Caro $\mathrm{R}$ and Artibani W: Preoperative Aspects and Dimensions Used for an Anatomical (PADUA) Classification of renal tumours in patients who are candidates for nephron-sparing surgery. Eur Urol 56: 786-793, 2009.

4 Kutikov A and Uzzo RG: The R.E.N.A.L. nephrometry score: a comprehensive standardized system for quantitating renal tumor size, location and depth. J Urol 182: 844-853, 2009.

5 Desantis D, Lavallee LT, Witiuk K, Mallick R, Kamal F, Fergusson $\mathrm{D}$, Morash C, Cagiannos I and Breau RH: The association between renal tumour scoring system components and complications of partial nephrectomy. Can Urol Assoc J 9: 39-45, 2015.

6 Kutikov A, Smaldone MC, Egleston BL, Manley BJ, Canter DJ, Simhan J, Boorjian SA, Viterbo R, Chen DY, Greenberg RE and Uzzo RG: Anatomic features of enhancing renal masses predict malignant and high-grade pathology: a preoperative nomogram using the RENAL nephrometry score. Eur Urol 60: 241-248, 2011.
7 Nagahara A, Uemura M, Kawashima A, Ujike T, Fujita K, Miyagawa Y and Nonomura N: R.E.N.A.L. nephrometry score predicts postoperative recurrence of localized renal cell carcinoma treated by radical nephrectomy. Int J Clin Oncol 21: 367-72, 2016.

8 Simmons MN, Hillyer SP, Lee BH, Fergany AF, Kaouk J and Campbell SC: Nephrometry score is associated with volume loss and functional recovery after partial nephrectomy. J Urol 188 : 39-44, 2012.

9 Canter D, Kutikov A, Manley B, Egleston B, Simhan J, Smaldone M, Teper E, Viterbo R, Chen DYT, Greenberg RE and Uzzo RG: Utility of the R.E.N.A.L.-nephrometry scoring system in objectifying treatment decision-making of the enhancing renal mass. Urology 78: 1089-1094, 2011.

10 Heidenreich A and Ravery V: Preoperative imaging in renal cell cancer. World J Urol 22: 307-315, 2004

11 Johnson PT, Horton KM and Fishman EK: How not to miss or mischaracterize a renal cell carcinoma: Protocols, pearls, and pitfalls. Am J Roentgenol 194: W307-315, 2010.

12 Clavien PA, Barkun J, de Oliveira ML, Vauthey JN, Dindo D, Schulick RD, de Santibañes E, Pekolj J, Slankamenac K, Bassi C, Graf R, Vonlanthen R, Padbury R, Cameron JL and Makuuchi $\mathrm{M}$ : The Clavien-Dindo classification of surgical complications: five-year experience. Ann Surg 250: 187-196, 2009.

13 Mitropoulos D, Artibani W, Graefen M, Remzi M, Roupret M and Truss M: Reporting and grading of complications after urologic surgical procedures: an ad hoc EAU guidelines panel assessment and recommendations. Eur Urol 61: 341-349, 2012.

14 Moreno-Alarcón C, Ramírez-Backhaus M and Pelechano-Gómez $\mathrm{P}$, Ortiz-Rodríguez IM, Rubio-Briones J, Rodríguez-Torreblanca $\mathrm{C}$ and Solsona-Narbón E: Nephrometry scores: Interobserver reproducibility and perioperative factors prediction. Actas Urol Esp 38: 523-529, 2014 (English Edition).

15 Spaliviero M, Poon BY, Aras O, Di Paolo PL, Guglielmetti GB, Coleman CZ, Karlo CA, Bernstein ML, Sjoberg DD, Russo P, Touijer KA, Akin O and Coleman JA: Interobserver variability of R.E.N.A.L., PADUA, and centrality index nephrometry score systems. World J Urol 33: 853-858, 2015.

16 Benadiba S, Verin A-L, Pignot G, Bessede T, Drai J, Bahi R, Toussile W, Rocher L and Patard J-J: Are urologists and radiologists equally effective in determining the RENAL Nephrometry score? Ann Surg Oncol 22: 1618-1624, 2015.

17 Silverman SG, Leyendecker JR and Amis ES: What is the current role of CT urography and MR urography in the evaluation of the urinary tract? Radiology 250: 309-323, 2009.

18 Rouprêt M, Babjuk M, Compérat E, Zigeuner R, Sylvester RJ, Burger M, Cowan NC, Gontero P, Van Rhijn BWG, Mostafid AH, Palou J and Shariat SF: EAU guidelines on urothelial carcinoma of the upper urinary tract: 2017 Update. Eur Urol 73: 111-122, 2018.

19 Tomaszewski JJ, Smaldone MC, Mehrazin R, Kocher N, Ito T, Abbosh P, Baber J, Kutikov A, Viterbo R, Chen DY, Canter DJ and Uzzo RG: Anatomic complexity quantitated by nephrometry score is associated with prolonged warm ischemia time during robotic partial nephrectomy. Urology 84: 340-344, 2014.

20 Vilaseca RM, Westphalen AC, Reis HF, Zogbi OS, Silva GE, Dos Reis RB and Muglia VF: Reproducibility and interobserver agreement of the R.E.N.A.L. nephrometry score: focus on imaging features. Radiol Bras 50: 7-12, 2017.

Received March 13, 2018

Revised March 31, 2018

Accepted April 3, 2018 